\title{
Violencia basada en género en zonas urbanas y urbano-marginales de Lima y Callao, 2007 - 2010
}

Gender-based violence in Lima and Callao's urban areas and urban fringe 2007 - 2010

\author{
Pedro Mascaro-Sánchez ${ }^{1,2}$, Susana García-Parra ${ }^{3, a}$, Óscar Munares-García ${ }^{4, a}$ \\ 1 Director General, Instituto Nacional Materno Perinatal, Lima, Perú. \\ ${ }^{2}$ Profesor Principal, Facultad de Medicina, Universidad Nacional Mayor de San Marcos, Lima, Perú. \\ ${ }^{3}$ Profesora Asociada de la Facultad de Medicina de la UNMSM. \\ ${ }^{4}$ Profesor Auxiliar de la Facultad de Medicina de la UNMSM. \\ ${ }^{a}$ Licenciada en Obstetricia del INMP.
}

\begin{abstract}
Resumen
Objetivos: Determinar y comparar las características de la violencia en pobladores de zonas urbanas y urbano-marginales. Diseño: Estudio epidemiológico descriptivo. Institución: Escuela de Obstetricia, Facultad de Medicina, Universidad Nacional Mayor de San Marcos, Lima, Perú. Participantes: Mujeres de Lima y Callao. Intervenciones: Entre los años 2007 y 2010, se aplicó una entrevista estructurada a 3971 mujeres de zonas urbanas y urbano-marginales de Lima y Callao. Se incluyó mujeres residentes de la zona, excluyéndose aquellas con problemas psicológicos evidentes y problemas del habla. Los datos fueron analizados con estadisticas descriptivas y con el estadístico chi cuadrado, significativo a un nivel $p<0,05$. Principales medidas de resultados: Violencia física. Resultados: De las entrevistadas, 39\% tenía entre 20 y 24 años de edad. El 47\% identificó violencia física, siendo similar en zonas urbanas y urbano-marginales. Los empujones y bofetadas fueron la forma más frecuente de violencia en ambas áreas. Con respecto al estudio de la violencia psicológica, fueron más frecuentes la utilización de gritos y celos por posesividad, también en ambas áreas. El gasto de la pareja sin la consulta fue ligeramente mayor para el área urbano marginal (49\%) que para el área urbana (42\%). El intento de tener relaciones sexuales contra su voluntad fue el tipo de violencia más frecuente (37\%), más en el área urbana (41\%) que en el área urbano marginal (34\%). Conclusiones: Se evidenció episodios de violencia física, psicológica, económica y sexual similares para el área urbana y el área urbano-marginal, siendo la violencia sexual más frecuente en el área urbana.

Palabras clave: Violencia, violencia doméstica, violencia contra la mujer, violencia sexual, población suburbana, población urbana.

\section{Abstract}

Objectives: To determine and compare gender-based violence characteristics in urban populations and urban fringe. Design: Comparative cross-sectional prospective study. Setting: School of Obstetrics, Faculty of Medicine, Universidad Nacional Mayor de San Marcos, Lima, Peru. Participants: Women from Lima and Callao. Interventions: Between 2007 and 2010 a structured interview was applied to 3971 women from urban areas and marginal urban areas of Lima and Callao. Women included were local residents, and women with obvious psychological problems and speech problems were excluded. Data was analyzed with descriptive statistics and chi-square method, with significant level $p<0,05$. Main outcome measures: Violence. Results: Thirty-nine per cent of respondents were between 20 to 24 years of age; $47 \%$ identified physical violence, similar at urban and marginal urban areas. Pushing and slapping were the most frequent violence forms at both areas. Psychological violence consisted in shouting, possessiveness and jealousy at both urban and marginal urban areas. Spending without consulting the couple was slightly higher at marginal urban areas (49\%) than in urban areas (42\%). Attempt to have sex against will was the most frequent type of violence (37\%), more in the urban area $(41 \%)$ than in marginal urban areas (34\%). Conclusions: Episodes of physical, psychological, economic, and sexual violence episodes were similar for urban and urban fringe areas, but marginal sexual violence was more frequent in the urban area.
\end{abstract}

Keys words: Violence, domestic violence, violence against women, sexual violence, suburban population, urban population.

An Fac med. 2012;73(2):127-33

\section{INTRODUCCIÓN}

La violencia constituye un problema de salud y un importante factor de riesgo psicosocial, por la magnitud del daño, invalidez y muerte que puede provocar, con consecuencias múltiples en el nivel social, psicológico y biológico. Ello, a pesar de no constituir una enfermedad en el sentido tradicional, donde el elemento etiológico-biológico desempeña como regla un papel fundamental ${ }^{(1)}$.
La información sobre la violencia hacia las mujeres es escasa y varía según el diseño de los estudios y los países donde estos son realizados. En una revisión de 26 trabajos procedentes de 20 países realizada por Heise, entre 20 y $75 \%$ de las mujeres había sufrido actos de violencia. El estudio reveló grandes diferencias, no solo según el país, sino también según el tipo de población estudiada urbana o rural ${ }^{(2)}$. El fenómeno de la violencia en América Latina ha sido relacionado con el aumento de la exclusión social, política y económica que experimenta una gran parte de la población. Asimismo, con la debilidad institucional de los estados y con la calidad baja de la democracia. La ENDES 2004-2005 determinó una prevalencia de violencia de 9,8\%, la Organización Mundial de la Salud (OMS) (Lima, 2002) $22,5 \%$, mientras que los datos del Ministerio de la Mujer y Desarrollo Social (MINDES) indican 28,6\% 
(Ayacucho, 2005) (3). En el Perú, dos mujeres son agredidas físicamente cada minuto, un millón habría sido agredida por su esposo o compañero al año, 27 mujeres son forzadas por su pareja a tener relaciones sexuales cada hora y 230 mil mujeres son forzadas sexualmente al año ${ }^{(4)}$.

Los diferentes grados, niveles y concepciones de la violencia están en correspondencia con los valores, normas y creencias de cada país, época y clase social. La familia como célula fundamental de la sociedad no está exenta de la práctica de violencia y la violencia intrafamiliar es considerada como una forma de violencia social ${ }^{(5)}$. En el $64 \%$ de los casos de violencia intrafamiliar hay antecedentes de violencia en la familia, por lo que se plantea el peligro que tiene la transmisión generacional, al igual que se afirma que mientras más violencia reciba un niño de sus padres, más proclive es este a ser violento con otros durante su adultez ${ }^{(6)}$.

Aproximadamente $20 \%$ de las mujeres que consultan en los servicios sanitarios padece de violencia doméstica. A pesar de que los servicios sanitarios son un punto clave para el abordaje de este tipo de violencia, rara vez los profesionales sospechan la existencia de esta situación y solo se identifica un $1 \%$ del total estimado de casos de mujeres maltratadas. El porcentaje bajo de profesionales que consideran que se trata de un problema de salud, la escasa consideración del papel de los servicios médicos ante este problema y las pocas personas que acuden por este problema al hospital, contrastan con los resultados que indican que aproximadamente $20 \%$ de las mujeres que acuden al médico padece algún tipo de violencia doméstica, así como, con la percepción que de este problema tienen los profesionales de otros países ${ }^{(7)}$.

La violencia contra la mujer es cualquier acción o conducta basada en el género que cause muerte, daño o sufrimiento físico, sexual o psicológico, tanto en el ámbito público como en el privado. Se trata de un problema que afecta los derechos humanos de las mujeres y un importante problema de salud pública en todo el mundo.

La violencia física es considerada como toda lesión física o corporal que deja huellas o marcas visibles; esta incluye golpes, bofetadas, empujones, entre otras. El $41 \%$ de las mujeres alguna vez ha sido empujada, golpeada o agredida físicamente por su esposo o compañero. Los estudios nacionales indican que $14 \%$ de las víctimas ha requerido atención en un establecimiento de salud ${ }^{(1)}$.

La violencia psicológica se refiere al hostigamiento verbal entre los miembros de la familia a través de insultos, críticas permanentes, descréditos, humillaciones, silencios, entre otras; es la capacidad de destrucción con el gesto, la palabra y el acto. Actitudes que tienen por objeto causar temor, intimidar, y controlar las conductas, sentimientos y pensamientos de la persona a quién se está agrediendo, como las descalificaciones, insultos o control. Esta no deja huellas visibles inmediatas, pero sus implicaciones son más trascendentes luego.

La violencia económica se refiere a privar de los recursos necesarios para subsistir a aquellas personas por las cuales no tendría que hacerlo la persona responsable del mismo; entre ellos tenemos el no cubrir las necesidades básicas y ejercer control a través de recursos económicos, gastos de pareja sin consulta previa, exigir explicaciones por uso del dinero, destinar menos dinero a la pareja a pesar de contarlo, justificar no ofrecer más recursos sugeridos por pareja, propiedades conyugales a nombre de uno de los miembros, disposición de dinero o herencia por una persona y privar de los medios de subsistencia mínimos para las personas, como la vestimenta, comida, vivienda o movilidad, a pesar de contar con las posibilidades.

La violencia sexual es un abuso basado en el género, según se establece en la Declaración para la Erradicación de todas las Formas de Discriminación contra la Mujer, y es frecuentemente perpetrada por un varón en el que la mujer confía y de quien espera protección, como el padre, el tío, la pareja, entre otros. De esta forma se puede decir que una de cada tres mujeres entrevistadas ha sufrido alguna forma de violencia sexual en su vida ${ }^{(8-10)}$.

Se denomina zonas urbanas marginales a las zonas donde las viviendas son aún precarias, no se cuenta o se cuenta de forma parcial con los servicios básicos de agua, desagüe, luz y comunicaciones adecuadas. Generalmente, estas zonas son ocupadas por migrantes, personas con pocas posibilidades económicas o donde los servidores públicos no han atendido las necesidades básicas ${ }^{(13)}$. El crecimiento de las ciudades es visto como un impulso al desarrollo, pero también como un incremento de los bolsones de pobreza, marginalidad y desigualdad. La existencia de violencia en las ciudades no puede entenderse por una sola causa. Algunos estudios han identificado ciertos factores relacionados a la existencia de violencia urbana; el tamaño, la densidad y la edad de la población parecerían apuntar a un incremento de la violencia. En este sentido, los principales factores que ayudan a interpretar la violencia urbana serían de orden demográfico. Según algunos estudios, si más de $40 \%$ de la población tiene entre 15 y 30 años, la posibilidad de un brote de violencia urbana se incrementa ${ }^{(11,12)}$.

Por lo antes expuesto, planteamos comparar las características de la violencia (física, psicológica, económica y sexual) en pobladores de zonas urbanas y urbanas marginales de Lima y Callao, durante un periodo de 4 años.

\section{MÉTODOS}

El presente fue un estudio epidemiológico descriptivo, con un componente de análisis en forma prospectiva y transversal. Comparó un grupo de mujeres de zonas urbanas y urbano-marginales, tanto de Lima como del Callao, realizado entre los años 2007 y 2010, con 
el apoyo de las profesoras y alumnos de obstetricia comunitaria de la Universidad Nacional Mayor de San Marcos. La muestra correspondió a 3971 mujeres de Lima (29 zonas) y Callao (9 zonas), integrada por 1542 de zonas urbano marginales y 2429 de zonas urbanas.

Los criterios de selección fueron residir en la zona, estar dispuesta a ser entrevistada, excluyéndose a las personas con dificultades psicológicas y del hablar evidentes y a los varones.

Las variables estudiadas fueron: a) la zona de residencia (urbana - urbano marginal); b) características generales: edad, estado civil, grado de instrucción, procedencia, ocupación; c) violencia física (Sí-No), actos de violencia realizados, frecuencia de hechos, denuncia de los hechos y solicitó atención de servicios de salud; d) violencia psicológica: tipos, afectación de los actos en personalidad; e) violencia económica: destino del dinero, justificación del gasto, gastos sin consultar, destino de los recursos, privación de necesidades básicas, explicaciones para uso del dinero y manejo de la propiedad; $y, f$ ) violencia sexual: intento de relaciones sexuales contra su voluntad, inconsciente, forzada sin protección, con agresiones físicas, relaciones sexuales anales, visualizar pornografía, con introducción de objetos y obligada a visualizar relaciones sexuales de pareja con otra. Los datos obtenidos pertenecieron a la historia de violencia y no a actos de violencia ocurridos en el momento de la entrevista. Para recoger la información se confeccionó y aplicó un formulario con las variables especificadas.

Durante el periodo de investigación se capacitó a estudiantes de la Escuela de Obstetricia de la Universidad $\mathrm{Na}$ cional Mayor de San Marcos, los cuales se desplazaron en grupos para la aplicación de las entrevistas. Se procedió a identificar las zonas urbanas y urbanomarginales previstas, según acuerdo entre alumnos y coordinador. Luego, se procedió al muestreo por manzanas en las zonas respectivas. Se solicitaba el permiso a las personas en la vivien- da, para ubicar a las mujeres con pareja estable. Después del consentimiento verbal, se procedió a aplicar el instrumento de recolección de datos en un tiempo de 28 minutos. Finalmente, se agradecía por la participación.

Para el análisis, se confeccionó una base de datos en Microsoft Excel, se aplicó estadísticas descriptivas, distribución de frecuencias y porcentajes. Para las inferencias estadísticas se aplicó el estadístico chi cuadrado, a un nivel de significación estadístico $\mathrm{p}<0,05$.

\section{RESULTADOS}

Con respecto a las características generales, la mayoría de los encuestados se encontraba en el rango de edad entre 20 y 24 años (39\%), siendo similar para los que habitaban en áreas urbanas como en áreas rurales. La mayoría era conviviente, dependiendo del área rural o urbana. La procedencia de las participantes fue de la costa $(48 \%)$, pero hubo diferencias en las áreas urbana y rural. La mayoría de quienes residían en el área urbana procedía de la costa

Tabla 1. Características generales de los participantes, según zona de residencia.

\begin{tabular}{|c|c|c|c|}
\hline \multirow[b]{2}{*}{ Características generales } & \multicolumn{2}{|c|}{ Zona de residencia } & \multirow[b]{2}{*}{$\begin{array}{c}\text { Total } \\
\mathrm{n}=3971 \\
\%\end{array}$} \\
\hline & $\begin{array}{c}\text { Urbana } \\
\mathrm{n}=2429 \\
\%\end{array}$ & $\begin{array}{c}\text { Urbano-marginal } \\
\mathrm{n}=1542 \\
\%\end{array}$ & \\
\hline \multicolumn{4}{|l|}{ Edad (años) } \\
\hline 15 a 19 & 6 & 13 & 10 \\
\hline 20 a 24 & 34 & 20 & 26 \\
\hline 25 a 35 & 35 & 42 & 39 \\
\hline más 35 & 24 & 25 & 25 \\
\hline \multicolumn{4}{|l|}{ Estado civil } \\
\hline Soltera & 19 & 20 & 20 \\
\hline Casada & 25 & 24 & 25 \\
\hline Conviviente & 48 & 47 & 48 \\
\hline Divorciada & 7 & 6 & 6 \\
\hline Otro & 1 & 2 & 1 \\
\hline \multicolumn{4}{|l|}{ Grado de instrucción } \\
\hline Ninguno & 2 & 7 & 4 \\
\hline Primaria & 21 & 22 & 21 \\
\hline Secundaria & 56 & 51 & 53 \\
\hline Superior técnica & 16 & 13 & 14 \\
\hline Superior universitaria & 6 & 8 & 7 \\
\hline \multicolumn{4}{|l|}{ Procedencia } \\
\hline Costa & 56 & 40 & 48 \\
\hline Sierra & 33 & 47 & 40 \\
\hline Selva & 10 & 13 & 12 \\
\hline \multicolumn{4}{|l|}{ Ocupación } \\
\hline Comerciante & 15 & 13 & 14 \\
\hline Ama de casa & 61 & 52 & 55 \\
\hline Obrera & - & 1 & 1 \\
\hline Técnica & 2 & 2 & 2 \\
\hline Profesional & 1 & - & - \\
\hline Otro & 21 & 32 & 28 \\
\hline Total & 100 & 100 & 100 \\
\hline
\end{tabular}


(56\%), mientras que las que residían en el área urbano-marginal, procedía mayoritariamente de la sierra (47\%) (tabla 1).

Al realizar el análisis comparativo, podemos apreciar que, en las zonas urbanas, la violencia más frecuente era la violencia física (46\%), seguida de la económica $(42 \%)$ y la sexual $(41 \%)$; mientras que en zonas urbano marginales la más frecuente fue la violencia económica (52\%), luego la física (48\%), la sexual (34\%) y la psicológica (21\%). Las diferencias fueron significativas, excepto en la violencia física (tabla 2).

Con respecto a los actos de violencia, los empujones y bofetadas fueron los más frecuentes en ambas áreas estudiadas. Estos hechos se habían repetido
Tabla 2. Distribución de los tipos de violencia, según zona de residencia.

\begin{tabular}{ccccccc} 
& \multicolumn{5}{c}{ Población } & \\
\cline { 2 - 3 } Tipo de violencia & \multicolumn{2}{c}{$\begin{array}{c}\text { Urbana } \\
(n=2429)\end{array}$} & & \multicolumn{2}{c}{$\begin{array}{c}\text { Urbano-marginal } \\
(n=1542)\end{array}$} & \\
\cline { 2 - 3 } & Número & $\%$ & & Número & $\%$ & \\
Económica & 1020 & 42 & & 802 & 52 & $<0,001$ \\
Física & 1117 & 46 & & 648 & 48 & 0,180 \\
Sexual & 996 & 41 & & 524 & 34 & $<0,001$ \\
Psicológica & 581 & 24 & & 324 & 21 & $<0,001$ \\
\hline Total & 3714 & 153 & 2298 & 155 & \\
\hline
\end{tabular}

Nota: Muchas mujeres pueden ser víctimas de diferentes tipos de violencia, por eso es que la suma de las frecuencias parciales de cada tipo supera el $100 \%$. tres a más veces. Más de la mitad de las participantes indicó que no denunció estos hechos, siendo ligeramente mayor para las del área urbana (53\%) que para las del área urbana marginal
Tabla 3. Violencia física según zona de residencia.

\begin{tabular}{|c|c|c|c|c|c|}
\hline \multirow{2}{*}{ Violencia física } & \multicolumn{2}{|c|}{ Zona de residencia } & \multirow[b]{2}{*}{$\begin{array}{c}\text { Total } \\
\mathrm{n}=3971 \\
\%\end{array}$} & \multirow[b]{2}{*}{$\mathrm{X}^{2}$} & \multirow[b]{2}{*}{$\mathrm{p}$} \\
\hline & $\begin{array}{c}\text { Urbana } \\
n=2429 \\
\%\end{array}$ & $\begin{array}{c}\text { Urbano- } \\
\text { marginal } \\
\mathrm{n}=1542 \\
\%\end{array}$ & & & \\
\hline \multicolumn{6}{|c|}{ Actos de violencia realizados } \\
\hline Empujones & 32 & 34 & 33 & & \\
\hline Bofetadas & 24 & 25 & 25 & & \\
\hline Patadas & 13 & 11 & 12 & & \\
\hline Puñetazos & 9 & 10 & 10 & & \\
\hline Le arrojaron objetos & 8 & 10 & 9 & & \\
\hline Otro * & 13 & 11 & 10 & & \\
\hline \multicolumn{6}{|c|}{ Estos hechos se repitieron } \\
\hline Varias veces & 27 & 4 & 12 & & \\
\hline Ninguna & 11 & 3 & 6 & & \\
\hline Una vez & 19 & 36 & 30 & & \\
\hline 2 veces & 19 & 23 & 22 & & \\
\hline 3 a más & 25 & 34 & 31 & & \\
\hline \multicolumn{6}{|l|}{ Lo denunció } \\
\hline No & 53 & 50 & 51 & & \\
\hline A familiares & 25 & 27 & 26 & & \\
\hline A vecinos & 5 & 11 & 8 & & \\
\hline A la policía & 17 & 12 & 14 & 2,48 & 0,115 \\
\hline \multicolumn{6}{|c|}{ Solicitó atención en un servicio de salud } \\
\hline Sí & 28 & 21 & 24 & & \\
\hline No & 72 & 79 & 76 & 17,3 & $<0,001$ \\
\hline Total & 100 & 100 & 100 & & \\
\hline
\end{tabular}

* Heridas por arma, estrangulamiento, abandono en lugares peligrosos, no ayuda por enfermedad o herida, la sujetaron.
(50\%). También, la mayoría no solicitó los servicios de salud (76\%), siendo las cifras ligeramente mayores para el área urbano marginal (79\%) que para el área urbana $(72 \%)$, con diferencia estadísticamente significativa (tabla 3 ).

Con relación al estudio de la violencia psicológica, se identificó como los elementos más importantes la utilización de gritos, celos por posesividad, los insultos y críticas como los más frecuentes, tanto para el área urbana como para el área urbano marginal, con diferencia estadísticamente significativa. La mayoría indicó que estos actos afectaban su personalidad, siendo similar para el área urbana como para el área urbana marginal (60\% y 63\%, respectivamente) (tabla 4).

En violencia económica, se pudo identificar que el evento más resaltante fue el gasto de la pareja sin la consulta del mismo (45\%), siendo ligeramente mayor para el área urbana marginal (49\%) que para el área urbana (42\%); así mismo, el exigir explicaciones por el uso del dinero (44\%), mayor para el área urbana marginal (52\%) que para el área urbana (38\%), con diferencia estadísticamente significativa (tabla 5).

El intento de relaciones sexuales contra su voluntad, el tener relaciones sexuales inconscientes o tener miedo a negarse y forzar el acto sexual fueron los tipos de violencia sexual más frecuentes. El intento de tener relaciones sexuales contra su voluntad fue el más frecuente $(37 \%)$, más frecuente en el área urbana (41\%) que en el área ur- 
Tabla 4. Violencia psicológica según zona de residencia.

\begin{tabular}{|c|c|c|c|c|c|}
\hline \multirow[b]{2}{*}{ Efectos de violencia psicológica } & \multicolumn{2}{|c|}{ Zona de residencia } & \multirow[b]{2}{*}{$\begin{array}{c}\text { Total } \\
\mathrm{n}=3971 \\
\%\end{array}$} & \multirow[b]{2}{*}{$X^{2}$} & \multirow[b]{2}{*}{$p$} \\
\hline & $\begin{array}{c}\text { Urbana } \\
\mathrm{n}=2429 \\
\%\end{array}$ & $\begin{array}{c}\text { Urbano- } \\
\text { marginal } \\
\mathrm{n}=1542 \\
\%\end{array}$ & & & \\
\hline \multicolumn{6}{|l|}{ Tipos } \\
\hline Gritos & 24 & 21 & 22 & & \\
\hline Celos, posesividad & 11 & 9 & 10 & & \\
\hline Frecuentes mentiras & 10 & 9 & 9 & & \\
\hline Insultos y críticas & 9 & 9 & 9 & & \\
\hline Acusaciones sin fundamento & 7 & 7 & 7 & & \\
\hline Rompimiento de promesas & 7 & 7 & 7 & & \\
\hline Degradación y humillación & 6 & 7 & 7 & & \\
\hline Culpas por todo lo que pasa & 5 & 7 & 6 & & \\
\hline Amenaza de daño & 5 & 7 & 6 & & \\
\hline Intimidación & 3 & 5 & 4 & & \\
\hline Aislamiento social & 4 & 3 & 3 & & \\
\hline Acciones destructivas & 3 & 4 & 3 & & \\
\hline Ridiculizar necesidades familiares & 3 & 3 & 3 & & \\
\hline Otro & 2 & 2 & 1 & 10,64 & $<0,001$ \\
\hline \multicolumn{6}{|l|}{ Estos actos afectan su personalidad } \\
\hline Sí & 60 & 63 & 61 & & \\
\hline No & 40 & 37 & 39 & 2,48 & 0,115 \\
\hline Total & 100 & 100 & 100 & & \\
\hline
\end{tabular}

bano marginal (34\%). El tener miedo al negarse a tener relaciones sexuales fue el segundo en frecuencia, siendo ligeramente mayor para el área urbana (17\%) que en el urbano marginal (15\%), con diferencia estadísticamente significativa (tabla 6).

\section{DISCUSIÓN}

La información sobre la violencia hacia las mujeres es escasa. En una revisión de 26 trabajos procedentes de 20 países realizada por Heise ${ }^{(2)}$, entre 20 y $75 \%$ de las mujeres habían sufrido actos de violencia; no contamos con estudios que hayan hecho este tipo de análisis comparando el área de residencia.

En el estudio presentado, se identificó $47 \%$ de episodios de violencia física, siendo las cifras similares tanto para el área urbana como para el área urbano- marginal. El informe de violencia contra la mujer identificó $24 \%$ de casos de violencia en la ciudad metropolitana de Lima y Callao. Los datos de ENDES (3) tuvieron resultados entre $41,2 \%$ (2000) y hasta 54,5\% (MINDES, 2005) para la violencia física, muy superiores a lo encontrado por nosotros. Frente a este problema, no buscar ayuda es un comportamiento similar en la costa, sierra y selva ${ }^{(10)}$. Centoni y Ferrin ${ }^{(15)}$ identificaron que el proceso de no buscar ayuda pueda deberse a que las víctimas de violencia no encuentran respuestas o seguridad con las personas o instituciones en las cuales solicitan ayuda. Almenares y colaboradores, en Cuba, en un estudio con 818 familias constataron que por cada 10 familias de la comunidad 1,2 (12\%) evidenciaron violencia intrafamiliar. El 56\% de las familias presentó problemas en cuanto a su dinámica relacional sistémica, familias con dificultades en la comunicación, cohesión, estructura de poder, dificultades para vivenciar y demostrar emociones positivas, y para cumplir funciones y responsabilidades negociadas en el núcleo familiar.

Con respecto a los actos de violencia en nuestro estudio, los empujones y bofetadas fueron los más frecuentes, tanto para el área urbana como para el área urbana marginal. Estos hechos dijeron se habían repetido tres a más veces. Los estudios nacionales indican que 14\% de las víctimas ha requerido atención en un establecimiento de salud; dos de cada cinco mujeres (40\%) refieren acudir a alguien para contarle su problema, pero, solo una de cada 10 (10\%) acude finalmente a una institución ${ }^{(1)}$. Almenares ${ }^{(5)}$ encontró que la violencia física observada en 34\% se expresó por bofetadas, empujones, puñetazos y golpizas, con o sin objetos; igualmente, se identificó en $12 \%$ de las familias el descuido de las necesidades de alimentación, abrigo o cuidados médicos. Más de la mitad en nuestro estudio indicó que no denunció estos hechos, siendo ligeramente mayor para las del área urbana (53\%) que para las del área urbano-marginal (50\%). La mayoría señaló no haber solicitado servicios de salud $(76 \%)$, siendo las cifras ligeramente mayores para el área urbano-marginal (79\%) que para el área urbana (72\%). Una de cada cuatro mujeres busca ayuda en la comisaría cuando es víctima de violencia familiar, siendo este el servicio al que acude el mayor número de mujeres; sin embargo, solo 38\% se siente satisfecha con el servicio brindado ${ }^{(10)}$. Otro estudio encontró que a pesar de que los servicios sanitarios son un punto clave para el abordaje de este tipo de violencia, rara vez los profesionales sospechan la existencia de esta situación y solo se identifica un 1\% del total estimado de casos de mujeres maltratadas.

Respecto a los diferentes tipos de violencia intrafamiliar, se apreció violencia psicológica en $90 \%$ de las familias estudiadas, caracterizándose por el hostigamiento verbal, gritos, insultos, amenazas, privación del saludo; los 
Tabla 5. Violencia económica según zona de residencia.

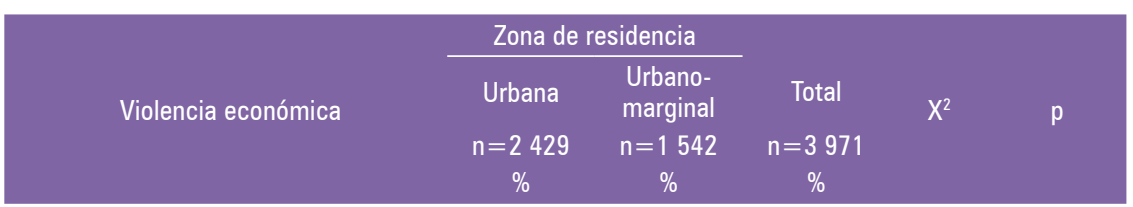

Gastos de pareja sin consultar

$\begin{array}{llllll}\text { Sí } & 42 & 49 & 45 & & \\ \text { No } & 58 & 51 & 55 & 16,1 & <0,001\end{array}$

Exigen explicaciones por uso de dinero

$\begin{array}{llllll}\text { Sí } & 38 & 52 & 44 & & \\ \text { No } & 62 & 48 & 56 & 74,6 & <0,001\end{array}$

Pareja destina menos dinero a pesar de contar con él

\section{Sí}

No

34

66

39

36

61

64

Justifica no recursos sugeridos por pareja

$\begin{array}{ll}\text { Sí } & 36 \\ \text { No } & 64\end{array}$

36

64

43

57

39

61

$16,9<0,001$

Propiedades conyugales a nombre solo del esposo

Sí

25
75

$\begin{array}{ll}40 & 31 \\ 60 & 69\end{array}$

31

69

$80,9<0,001$

Disposición de recursos producto sueldo 0 herencia

$\begin{array}{llllll}\text { Sí } & 18 & 38 & 27 & & \\ \text { No } & 82 & 62 & 73 & 166,4 & <0,001\end{array}$

Privación de vestimenta comida, vivienda y movilidad

\begin{tabular}{cccccc} 
Sí & 17 & 30 & 23 & & \\
No & 83 & 70 & 77 & 81,6 & $<0,001$ \\
\hline Total & 100 & 100 & 100 & & \\
\hline
\end{tabular}

elementos más importantes fueron los gritos, celos por posesividad, insultos y caricias, para ambas áreas, datos estadísticamente significativos. $\mathrm{Al}$ estudiar si estos actos afectaban su personalidad, la mayoría indicó que sí los afectaba, siendo similar tanto para el área urbana como para el área urbano-marginal (60\% y 63\%, respectivamente). Los diferentes grados, niveles y concepciones de la violencia están en correspondencia con los valores, normas y creencias de cada país, época y clase social. La familia como célula fundamental de la sociedad no está exenta de la práctica de violencia. Se señala que en $64 \%$ parejas jóvenes; esto también se refleja por el manejo de los recursos económicos. Resulta evidente que los porcentajes son altos para ambas áreas, indicando así, que el problema no se debe a un asunto asociado a la escasez de recursos sino a los problemas de comunicación de pareja. Con respecto a la violencia sexual, el intento de relaciones sexuales contra su voluntad fue el más frecuente $(37 \%)$, siendo más en el área urbana (41\%) que en el área urbano-marginal (34\%); el tener miedo al negarse a tener relaciones sexuales fue el segundo en frecuencia, siendo ligeramente mayor para el área urbana (17\%) que para el área urbana marginal (15\%); estos datos fueron estadísticamente significativos. En nuestro país, la violación sexual es la que ha merecido la mayor atención, habiéndose desconocido las otras formas de violencia contra las mujeres y contra los y las adolescentes ${ }^{(12)}$. Un estudio realizado por Ramos y colaboradores ${ }^{(8)}$ encontró que de las mujeres entrevistadas 19\% señaló que al menos alguna vez en su vida había sido objeto de tocamientos sexuales en contra de su voluntad; $11 \%$ habían sido violadas, $5 \%$ forzadas a tocar los órganos sexuales de otra persona en contra de su voluntad. Cabe señalar que la mitad de estas violencias ocurrió antes de los 19 años. De esta forma podemos decir que una de cada tres mujeres entrevistadas había sufrido alguna forma de violencia sexual en su vida. Cifuentes y Betancourt ${ }^{(15)}$ hicieron una revisión de casos de violencia sexual, encontrando que los profesionales de la salud brindaron poco apoyo a las violentadas y estas sufren definitivamente secuelas negativas posterior a los procesos violentos. De todos los tipos de violencia resulta más impactante el ocurrido por la violencia sexual; pero, también queremos indicar que este proceso no nace solo, sino en un clima de violencia basada en género ${ }^{(14)}$, que lamentablemente está muy presente en nuestra sociedad latinoamericana.

En conclusión, con respecto a los datos de violencia física, se identificó 
Tabla 6. Violencia sexual según zona de residencia.

\begin{tabular}{ccccc} 
& \multicolumn{2}{c}{ Zona de residencia } & \\
\cline { 2 - 3 } Violencia sexual & Urbana & $\begin{array}{c}\text { Urbano- } \\
\text { marginal }\end{array}$ & Total \\
& $n=2429$ & $n=1542$ & $n=3971$ \\
Intento de RS contra su voluntad & $\%$ & $\%$ & $\%$ \\
Tener miedo a negarse a RS o RS inconsciente & 41 & 34 & 37 \\
Forzada a RS sin protección & 17 & 15 & 16 \\
Insultada durante RS & 15 & 15 & 15 \\
Acusada falsamente de RS con otros & 8 & 5 & 6 \\
Agresiones físicas durante el acto sexual & 6 & 9 & 8 \\
Forzada a RS anales & 4 & 9 & 7 \\
Obligada a visualizar pornografía & 4 & 7 & 5 \\
Le ha introducido objetos en RS & 3 & 4 & 3 \\
Visualizó RS de pareja con otras personas & 1 & 2 & 1 \\
\hline Total & - & 1 & 1 \\
\hline
\end{tabular}

RS: Relaciones sexuales.

que $47 \%$ presentó episodios de violencia, siendo las cifras similares tanto para el área urbana como para el área urbano-marginal. En la violencia psicológica, se identificó como los elementos más importantes la utilización de gritos, celos por posesividad y los insultos. En la violencia económica, el evento más resaltante fue el gasto de la pareja sin la consulta del mismo, siendo ligeramente mayor para el área urbano-marginal. Y con respecto a la violencia sexual, el intento de tener relaciones sexuales contra su voluntad fue significativamente más frecuente para el área urbana que para el área urbano-marginal.

\section{REFERENCIAS BIBLIOGRÁFICAS}

1. Araujo R, Díaz G. Un enfoque teórico-metodológico para el estudio de la violencia. Rev Cub Salud Pública. 2000;24(2):85-90.

2. Núñez $P$, Monge R, Grios C, Elzondo A, Rojas A La violencia física, psicológica, emocional y sexual durante el embarazo: riesgo reproductivo predictor de bajo peso al nacer en Costa Rica. Rev Panam Salud Pública. 2003;14(2):75-83.

3. Ministerio de la Mujer y Desarrollo Social. Estado de las investigaciones sobre violencia familiar y sexual en el Perú 2001 - 2005. Programa Nacional Contra la Violencia Familiar y Sexual. 2da. Ed. 2006.

4. Ministerio de la Mujer y Desarrollo Social. Plan Nacional contra la violencia hacia la mujer 2009 2015. Disponible en: http://www.mimdes.gob.pe/ attachments/322_PNCVHM_2009-2015.pdf.

5. Almenares M, Lauro I, Ortiz M. Comportamiento de la violencia intrafamiliar. Rev Cub Med Gen Integr. 1999;15(3):285-92
6. Muñiz M, Ferrer D, González J, Jiménez Y. Violencia intrafamiliar, su presentación en un área de salud. Rev Cub Med Integr. 2000;16(5):468-73.

7. Coll-Vinent B, Echevarría T, Farras U, Rodríguez D, Millá J y col. El personal sanitario no percibe la violencia doméstica como un problema de salud. Gac Sanit. 2008;22(1):7-10.

8. Ramos-Lira L, Saltijeral-Méndez MT, Romero-Mendoza M, Caballero-Gutiérrez MA, Martinez-Vélez NA. Violencia sexual y problemas asociados en una muestra de usuarias de un centro de salud. Salud Pública Mex. 2001;43:182-91.

9. Almenares M, Lauro I, Ortiz M. Comportamiento de la violencia intrafamiliar. Rev Cub Med Gen Integr. 1999;15(3):285-92.

10. Macassi I, Casaverde M. Violencia familiar, violencia sexual, aborto, derechos reproductivos. Informe 2003. Centro de la mujer Flora Tristán. Noviembre 2003.

11. Tedesco L. Violencia urbana: un desafío al fortalecimiento institucional. El caso de América Latina. Fundación para las relaciones internacionales y dialogo exterior. Documento de Trabajo 78. Febrero de 2009.

12. Torres J. Violencia en barrios en pobreza extrema urbano-marginales y las respuestas estatales. Centro de Estudios Sociales Padre Juan Montalvo. Disponible en: http://www.centrojuanmontalvo.org. do/spip/IMG/pdf/Violencia_en_la_gran_pobreza_2_. pdf Acceso 12-12-2011

13. Rojas M. La vivienda precaria urbana marginal y su relación con la salud de la población en el proceso de sostenibilidad. Un enfoque teórico para la estimación del riesgo y la vulnerabilidad. Disponible en: http://www.alapop.org/2009/images/PDF/ ALAP2004_389.pdf acceso 17-12-2011

14. Ortiz D, Távara L. Buenas prácticas para la atención de la violencia sexual contra las mujeres. Federación Latinoamericana de Sociedades de Obstetricia y Ginecología (FLASOG). 2011.

15. Távara L. Investigaciones e intervenciones sobre violencia sexual desarrollada en América Latina y el Caribe. Federación Latinoamericana de Sociedades de Obstetricia y Ginecología (FLASOG). 2011.

Artículo recibido el 9 de marzo de 2012 y aceptado para publicación el 5 de mayo de 2012.

Correspondencia:

Dr. Pedro Mascaro Sánchez

Correo electrónico: pmascaros@hotmail.com 